\title{
OVERVIEW OF BIOINFORMATICS AND MEDICAL INFORMATICS TECHNOLOGY FOR NON-SPECIALISTS
}

\author{
A. James Cuticchia
}

\section{A. DEFINITIONS AND DISTINCTIONS}

1. Definition and history of "bioinformatics" 1.01

2. Definition and history of "medical informatics"

3. Distinctions between bioinformatics and medical informatics

B. FUNDAMENTAL MOLECULAR BIOLOGY

1. DNA replication

2. mRNA transcription

3. Translation

\subsection{1}

1.02

1.03

1.04

1.05

1.06

1.07
C. TECHNOLOGIES OF BIOINFORMATICS AND

MEDICAL INFORMATICS 1.08

1. Next Generation Sequencing technology 1.09

2. Cancer genomics $\quad 1.10$

3. Semantics 1.11

D. GENOME-WIDE ASSOCIATION STUDIES $\quad 1.12$

1. i2b2 1.13

2. $\operatorname{tranSMART} 1.14$

E. MOVING FORWARD $\quad 1.15$

The following chapters of this book will discuss legal issues pertinent to bioinformatics and medical informatics. While some topics, such as intellectual property, are applicable to fields outside of bioinformatics and medical informatics as well, overall the following chapters will focus on application to these fields.

Since some readers may not be aware of the history and technologies of these disciplines, they may find this chapter useful. It will briefly discuss the history of each discipline and distinctions between bioinformatics and medical informatics, and will give an overview of the technologies related to them. 


\section{A. DEFINITIONS AND DISTINCTIONS}

\section{Definition and history of "bioinformatics"}

1.01 The definition of bioinformatics aptly fits the words which, in relation to another topic, Justice Potter Stewart stated, "I understand to be embraced with that shorthand description; and perhaps I could never succeed in intelligibly doing so. But I know it when I see it..."1

A Google Search in 2012 for [bioinformatics + definition:before 2013] yielded more than 400,000 results. That same search performed in 2020 yields more than 6 million results. ${ }^{2}$ The growth of results is a combination of bioinformatics' increased prevalence over the past seven years and its expansion. In any case, it is inarguable that the presence of bioinformatics on the World Wide Web has increased.

The early definition of bioinformatics has been attributed to Paulien Hogeweg and to Ben Hesper, whom many have recognized as the creators of bioinformatics. The purported first definition of the term was "the study of informatic processes in biotic systems."

While the origin has been traced to publications by these authors in 1978, Hogeweg claims that they were using the term "bioinformatics" as early as the beginning of the 1970 s. ${ }^{4}$ This definition was derived in part from the application of computers to biology, which became known as computational biology. Today both terms are used with slight distinctions.

A more modern definition of bioinformatics is " $[t]$ he application of tools of computation and analysis to the capture and interpretation of biological data." ${ }^{5}$

\section{Definition and history of "medical informatics"}

1.02 While bioinformatics evolved and expanded naturally from the work of researchers in the discipline, medical informatics took a slightly different route. The field of medical informatics organized into the American Medical Informatics Association early in its development. Their work in practice is roughly analogous to that of the American Bar Association in providing a forum for advocacy, initiatives, organization, and fellowship.

AMIA (the American Medical Informatics Association) is a professional scientific association that was formed by a merger of three organizations in 1988: the American Association for Medical Systems and Informatics; the American College of Medical Informatics; and the Symposium on Computer Applications in Medical Care. ${ }^{6}$ AMIA developed a formal definition of biomedical informatics in 2012: "Biomedical informatics (BMI) is the interdisciplinary field

1 Jacobellis v Ohio, 378 U.S. 184, 197 (1964) (Stewart, P., concurring).

2 Search performed Jul. 19, 2020.

3 Paulien Hogeweg, The Roots of Bioinformatics in Theoretical Biology, 7(3) PLOS Computational Biology https://doi.org/ 10.1371/journal.pcbi.1002021 (2011).

4 Ibid.

5 Ardeshir Bayat, Science, Medicine, and the Future: Bioinformatics, Clinical Rev. 1018 (2002).

6 AMLA History, www.amia.org/about-amia/mission-and-history (last visited Jul. 19, 2020). 
that studies and pursues the effective uses of biomedical data, information, and knowledge for scientific inquiry, problem solving, and decision making, driven by efforts to improve human health."

\section{Distinctions between bioinformatics and medical informatics}

Both disciplines continue to evolve. Thus, the definitions of both will no doubt continue to change over time. Bioinformatics is a term built to define a discipline that has been changing over the years, and its definition is more diverse than that of medical informatics. Bioinformatics is a field which was defined through a grass-roots effort.

Medical informatics, predominantly through AMIA, has taken more of a "top-down" approach by delimiting the field to: (a) translational bioinformatics; (b) clinical research informatics; (c) clinical informatics; (d) consumer health informatics; and (e) public health informatics. ${ }^{8}$ Looking over the medical informatics field, it is arguably defined to those works dealing with clinical services and health research.

The term "translational bioinformatics" derives from translational medicine, also referred to as "bench to bedside" and later expanded to "bench to bedside to community." Translational medicine is research undertaken specifically to improve health, in contrast to basic research. ${ }^{9}$

\section{B. FUNDAMENTAL MOLECULAR BIOLOGY}

This section is a primer on molecular biology. For those who have a grasp of this topic, it may easily be skimmed over or even skipped. However, to fully understand many of the technologies discussed in this book, some knowledge of molecular biology is crucial.

\section{DNA replication}

DNA contains the blueprint for the making of a cell, the fundamental unit of life. This information is stored in a structure, which is composed of an alphabet of four different "bases." These bases are: (a) adenine, (b) thymine, (c) cytosine, and (d) guanine. As DNA is double-stranded, it is composed by pairing the complementary bases (adenine with thymine; cytosine with guanine). Because of this pairing, each strand of DNA is complementary to its sister's strand.

For example, a DNA sequence might read:

\section{AAGTAGCCATCAGTGACT (Strand 1)}

TTCATCGGTAGTCACTGA (Strand 2)

7 Casimir A. Kulikowski, Edward H. Shortliffe, Leanne M. Currie, et al., Informatics and Specification of Core Competencies for Graduate Education in the Discipline, American Med. Informatics Assoc. 93 I-8 (2012).

8 Why Informatics, www.amia.org/why-informatics, (last visited Jul. 20, 2020).

9 See generally, Xiandong Wang, A New Vision of Definition, Commentary, and Understanding in Clinical and Translational Medicine, 1(1), 5 Clin. Transl. Med. (20 I 2), https://doi.org/10.1186/2001-1326-1-5. 
This pairing of two complimentary strands facilitates replication of the DNA. During replication, each strand will separate and new bases of DNA will be annealed to each strand:

\author{
AAGTAGCCATCAGTGACT (Strand 1) \\ TTCATCGGTAGTCACTGA (Strand 1 Copy) \\ TTCATCGGTAGTCACTGA (Strand 2) \\ AAGTAGCCATCAGTGACT (Strand 2 Copy)
}

\title{
2. mRNA transcription
}

1.06 Proteins are not made directly from DNA. In higher organisms the DNA is encased in a nuclear membrane within the cell. To create the working template for a protein, a copy of the DNA must transcribe mRNA (messenger RNA) created in the same manner as DNA is replicated. There are differences between DNA and mRNA. First, the sugar used for the backbones of RNA molecules is ribose, where DNA uses deoxy ribose. Second, in RNA the base thymine is replaced by uracil. Third, only one strand of the mRNA is exported through the nuclear membrane into the cell.

\section{Translation}

1.07 To produce a protein (for example, hemoglobin), the mRNA attaches itself to an organelle called the ribosome. Transfer RNA, tRNA, are matched with their complementary three-base code on the mRNA. Each tRNA carries with it an amino acid based on its complementary three-letter code. Therefore, when a tRNA matches the mRNA in the ribosome, an amino acid is added. The string of amino acids makes up the protein.

The working copy of the DNA is made by copying the one strand of the double-stranded helix with RNA. This molecule is identical in information, but is structurally different from the DNA molecule.

The process by which DNA $\rightarrow$ RNA $\rightarrow$ PROTEIN is called the "Central Dogma" of molecular biology. ${ }^{10}$

\section{TECHNOLOGIES OF BIOINFORMATICS AND MEDICAL INFORMATICS}

1.08 Both disciplines are based on four fundamental factors: (a) generating data; (b) warehousing data; (c) analyzing data; (d) providing results.

10 See generally, Gerald Bergtrom, Basic Cell and Molecular Biology (3 ed. 2018) https://open.umn.edu/opentextbooks/ textbooks/cell-and-molecular-biology-2e-what-we-know-how-we-found-out (discussing cellular science and molecular biology in detail, online as a CC-BY textbook). 
It is beyond the scope of this article to present all methods of generating and analyzing data. Therefore, only the major methods for generating the most common data will be discussed. Similarly, the focus will be on the most common informatics tools used in analysis and their results. Most of this topic will be common to both bioinformatics and medical informatics. Distinctions between those fields will be discussed where pertinent.

\section{Next Generation Sequencing technology}

The first complete human genome sequence was elucidated by an international effort which took 13 years and cost more than 1 billion dollars. During the project, improvements in sequencing methods and bioinformatics tools were created.

While the goal of the project was to map, sequence, and determine the functions of genes, the stretch goal was to be able to sequence an entire human genome quickly and at a cost of one thousand dollars or less. The main goal will likely not be achieved for some time, given the complexity of human genetics, but the stretch goal was. Determining the function of all human genes will be a task that will occupy genetic researchers for decades, and possibly more. However, a method of sequencing was developed shortly after the human genomic project was "completed," which accelerated the sequencing of human genomes in as short a period as one day at a cost of approximately one thousand dollars. This became possible through the process of Next Generation Sequencing (NGS).

While there are several NGS methods, Illumina sequencing is the most widely used. Here, we will provide a general overview of this form of sequencing.

DNA is first cleaved into smaller pieces ranging from 50 to 500 base pairs in length, and the strands are separated. Short sequences which are complementary to sequences on the sequencing chip are added (adapters). The sequencing chip has millions of DNA sequences that are complementary to the adapters. These ends bond the adapter, along with the DNA to which it was attached, to the sequencing plate. As there are two ends to each strand, the second end will attach to its complementary tree. This will allow the exposed DNA to be replicated. Using the polymerase chain reaction, a method for making multiple copies of sequences in repeated rounds of replication, each sequence is amplified multiple times in order to yield a sufficient amount of DNA to be read by the sequencer.

This is done using fluorescent dyes. Once the replicated strands of DNA have copy numbers sufficient for reading the sequence, fluorescent dye is applied to each of the four bases $(A, T$, $\mathrm{C}, \mathrm{G})$. The DNA sequences are then replicated with the dyed nucleotides, which are read in sequence by the sequencer, resulting in a sequencing read of the original cleaved DNA. ${ }^{11}$

Once the massive amount of data this provides is collected, the small sequence fragments must be assembled into a contiguous sequence(s). There are two ways to treat the computational power needed and error rate. The first is, for example, to cleave a human chromosome into tens

11 See Barton E. Slatco, Andrew F. Gardener, and Frederick M. Ausubel, Overview of Next Generation Sequencing Technologies, I22(I):E59 Curr Prot Mo Biol (Apr. 2008); A Beginner's Guide to NGS www.illumina.com/science/ technology/next-generation-sequencing/beginners.html (last visited Jul. 23, 2020). 
of millions of pieces and sequence. The second is to cleave all human chromosomes together and undertake a much more challenging process.

Sequences may be assembled more quickly if a reference sequence is available. While it is still necessary in some studies to sequence without a reference, it much more time-consuming and is only performed in specific circumstances, which will not be discussed here.

The first step of the analysis is to check the quality of the sequences. Errors can occur during the annealing with the fluorescent dye. Thus, built into the sequencers is a base calling algorithm. It presents a quality number for each base and assigns a score. Those that fall outside normal parameters are examined by a researcher.

Once the sequence is deemed to be of acceptable quality, the fragments are placed onto the complementary strand of the reference genome. Since many fragments will overlap both the reference genome and each other, a contiguous sequence is built.

In this technology, an overlap of bioinformatics and medical informatics exists. Both disciplines will use this process to determine a DNA sequence. In many cases this is done as a collaboration between researchers in both fields.

\section{Cancer genomics}

1.10 Precision medicine occurs where physicians base treatments, at least partially, on genetics. Twenty years ago, there was a three-step diagnosis and treatment plan. A tumor is found, the tumor and surrounding lymph nodes are removed, and a mixture of chemotherapy agents and/ or radiation is given.

Here, the oncologist passes the tumor sample for DNA sequencing (of specific regions, in many cases). The sequence is then returned to the oncologist, who consults the literature to see if there is any specialized treatment for a tumor with these genetics and, if so, evaluates it as a treatment option. The oncologist may choose this option (probably with adjuvant therapies) in the cancer treatment plan for the patient.

While some bioinformatics techniques are used, these procedures are considered by most to be medical informatics. ${ }^{12}$

\section{Semantics}

1.11 To combine patient records into usable data sets, it is imperative that representations of the data have the same name. For example, what is called "blood pressure" in one hospital could be named "BP" in another. Without the use of a standard nomenclature, medical informatics would hit a wall at the very start of the process.

12 See Precision Medicine in Cancer Treatment, www.cancer.gov/about-cancer/treatment/types/precision-medicine (last visited Jul. 2, 2020). 
There are two major systems which have been adopted in the medical informatics community. The first is SNOMED CT (Systemized Nomenclature of Medicine Clinical Terms). This system is the most widely used. The other is LOINC (Logical Information Identifiers, Names and Codes). SNOMED CT consists of 341,000 active concepts in the January 2018 release. ${ }^{13}$

The ICD should be familiar to all physicians. It is a dictionary for medical treatments and billing codes. This is being expanded to reflect the current state of medicine.

ICD-11 has been released to give countries time to prepare for endorsement at the World Health Assembly in 2019, aiming for global roll-out in 2022. It contains about 55,000 possible codes, with thousands of new classifications (some are truly for the modern age: the code $\mathrm{XE} 346$ can be used to indicate an injury inflicted by a spacecraft). Several novel features in ICD-11 are relevant to infectious diseases, the most important of which is in the coding of antimicrobial resistance, which has been greatly updated and expanded. ${ }^{14}$

SNOMED CT is not a list of terms which can be translated. It is based on concepts. Below is an example of how these concepts are used. ${ }^{15}$

\section{EXAMPLE: PRECOORDINATED REPRESENTATION OF "LAPAROSCOPIC EMERGENCY APPENDECTOMY"}

SNOMED CT contains the concept 174041007|laparoscopic emergency appendectomy|. The identifier of this concept (174041007) can be used (with or without the associated term) as a precoordinated expression to record an instance of this procedure. The procedure "laparoscopic emergency appendectomy" has at least three distinct facets: "removal of appendix", "using a laparoscope" as "emergency procedure." The SNOMED CT concept 174041007|laparoscopic emergency appendectomy| precoordinates these facets as its definition and includes the following defining relationships:

$$
\begin{aligned}
& 116680003 \mid \text { is a }|=80146002| \text { appendectomy } \mid \\
& 260870009 \mid \text { priority }|=25876001| \text { emergency } \mid \\
& 425391005 \mid \text { using access device }|=86174004| \text { laparoscope } \mid
\end{aligned}
$$

The majority - 78 percent — of mapped concepts have a single rule and single ICD-10 code. ${ }^{16}$ SNOMED CT is not being replaced by ICD-11. ICD-11, however, will have a built-in system to link to SNOMED terms. Thus, ICD codes can now be better used to define objects in SNOMED.

13 Oliver Bodenreider, Ronald Cornet, and Daniel J. Vreeman, Recent Developments in Clinical Terminologies-SNOWMED CT, LOINC, and RxNORM, 27(2) Yearb Med, Inform, I29-39 (Aug. 2018).

14 Editorial, 18 The Lancet www.thelancet.com/pdfs/journals/laninf/PIIS1473-3099(18)30436-5.pdf (last visited Jul. 25, 2020).

15 https://confluence.ihtsdotools.org/display/DOCSTART/7.+SNOMED+CT+Expressions (last visited Aug. 1, 2020).

16 James R. Campbell, Hazel Brear, Rita Schilone, et al., Semantic Interoperation and Electronic Health Records: Content Sensitive Mapping from SNOWMED CT to ICD-10, Stud Health Technol Inform, (2013), also available at https:// pubmed.ncbi.nlm.nih.gov/23920627/ (last visited Jul. 1, 2020). 


\section{GENOME-WIDE ASSOCIATION STUDIES}

1.12 Genome-Wide Association Studies (GWAS) utilize the fact that there is significant variability in the genome. This variability has been used in forensics to find DNA matches among individuals. GWAS uses the same principles to find variabilities which are linked to genetic traits or disease.

After the sequencing of the human genome, the HAPMAP project was undertaken to determine variability across the human genome. ${ }^{17}$ This variability was examined using single nucleotide polymorphisms (SNPs).

An SNP is a replacement of a single nucleotide with another (for example, A with a $G$ ) which occur in the non-coding region of DNA and do not affect the amino acid sequence. More than 100 million SNPs have been found in the human genome, with 4-5 million found in each person's genome. ${ }^{18}$

Presently, NGS is used to sequence DNA to look for SNPs. The power of the study is based on the number of samples taken. Usually these are taken from two groups. The first group of participants have a disease and the second group is a control.

SNPs only detect correlations. Correlations do not necessarily apply causation. A simple analogy here: people who ride rollercoasters eat ice cream-there is a correlation; but if a person eats ice cream the person does not necessarily ride rollercoasters.

SNPs can correlate with diseases without causing them. There are several SNPs which have an association with increased risk of heart disease. Four SNPs, though, are highly correlated to heart disease. While seven SNPs are common and generally of limited effect, one SNP correlates with a double risk of sudden cardiac death. ${ }^{19}$

SNPs can act as markers across a chromosome. Since the placement of a SNP is known, clusters can show areas which correlate with what trait or disease is being tested. Where SNPs fall inside known genes, this gives a target to a gene which correlates to a single-gene disease such as cystic fibrosis.

The term single nucleotide variant (SNV) is used more commonly than SNP in medical informatics. In essence, both terms refer to a single polymorphism (that is, variant) on a strand of DNA.

This work began solely as a bioinformatics endeavor, and is now an important component of medical informatics.

19 Heart Disease, SNPedia, https://www.snpedia.com/index.php/Heart_disease (last visited Jun. 2020). 


\section{1. $\mathrm{i} 2 \mathrm{~b} 2$}

Up to this point, technologies discussed have been those devoted to a single task or a single class of results. Informatics for Integrating Biology \& Bedside (i2b2) is one of the earliest to have considered a pipeline infrastructure of tools and databases to support the many aspects of medical informatics. It is open source and

[i] ts mission [is] to provide clinical investigators with the tools necessary to integrate medical record and clinical research data in the genomics age, a software suite to construct and integrate the modern clinical research chart. i2b2 software may be used by an enterprise's research community to find sets of interesting patients from electronic patient medical record data, while preserving patient privacy through a query tool interface. ${ }^{20}$

It is impossible to discuss much of the computer science used in this project, given the scope of this chapter. However, the fundamental principles of $\mathrm{i} 2 \mathrm{~b} 2$ which support medical informatics should suffice for that purpose.

One major focus is the creation of virtual datasets from clinical data and findings. The first step is to collect data, either from a single data warehouse or from the data available to the researcher. Private health information cannot be accessible to this system. Data from electronic medical records (EMR) are transformed to make them fit for combining datasets and better scientific analysis. ${ }^{21}$

An example of an analysis which can be performed through the web or workbench is determining the degree of linkage between autoimmune disease and heart disease. Both these diseases have many subtypes which can be pulled from a data warehouse. Computational tools which are integrated into $\mathrm{i} 2 \mathrm{~b} 2$ or available as stand-alone software can take this data, expand it, and create a workbench for further inquiry. Information such as patient age or onset of disease, cause and date of death, gender, and other data can be extracted.

If the researcher finds, for example, lupus having statistical correlation with the presence of surgery to replace heart valves, this can be looked at more broadly to see if patient demographics play a role, and so forth.

For the integration of genomic data, it is possible to perform this task with a variety of applications. As i2b2 and tranSMART have merged their nonprofit foundations, it makes sense to discuss this system and how it interoperates with $\mathrm{i} 2 \mathrm{~b} 2 .{ }^{22} \mathrm{~A}$ fuller discussion of tranSMART will follow this section.

There is no need to write additional software code within i2b2 to query both genomic and clinical data once the genomic data are loaded. ANNOVAR is a tool which is used to annotate variants and is a key step in the incorporation of genomic data into $\mathrm{i} 2 \mathrm{~b} 2$ or tranSMART. ${ }^{23}$

20 Shawn N. Murphy, Griffin Weber, Michael Mendis, et al., Serving the Enterprise and Beyond with Informatics for Integrating Biology and the Bedside (i2b2), 17(2) J. Am. Med. Inform. Assoc., 124-30 (Mar-Apr 2010).

21 Ibid.

$22 \mathrm{i} 2 \mathrm{~b} 2 /$ tranSmart Foundation, https://i2b2transmart.org (last visited Mar. 12, 2020).

23 Kai Wang, Mingyao Li, and Hakon Hakonarson, ANNOVAR: Functional Annotation of Genetic Variants from High-Throughput Sequencing Data, 38(16) Nucleic Acids Res (2ого), https://doi.org/10.1093/nar/gkq603. 
To perform this loading [into i2b2], we created a Perl script and associated Perl modules to divide the ANNOVAR input files into a set of text files that mimicked the structure of $\mathrm{i} 2 \mathrm{~b} 2$ 's schema. A first pass is made through the input files to gather the unique patient set and distinct attributes that are being observed about those patients. After this is done, unique identifiers are generated for each of the patients, as well as for the attributes obtained from the ANNOVAR-derived files. Once this is done, the script generates the intersection of these dimensions ...This approach of creating text files per database table and generating unique IDs externally allowed us to use Oracle's loading tool, SQL*Loader. The data are loaded directly into tables queried by the i2b2/tranSMART application. ${ }^{24}$

\section{2. $\operatorname{tranSMART}$}

1.14 There is some overlap in the functions of $\mathrm{i} 2 \mathrm{~b} 2$ and tranSMART. Both systems are open source, with public APIs and community-written extensions. TranSMART was initially created as an i2b2 spinoff in 2008 by a pharmaceutical company in the context of clinical trials; the tranSMART application added lightweight analytical capabilities, an expanded analysis framework, and other data management options. ${ }^{25}$

Like i2b2, tranSMART stores clinical data on patients. It also stores research data. An unexhaustive list of what researchers can do in the tranSMART system includes:

1. Compare data from proteomics, metabolomics, and other "omics" studies

2. Contrast patterns of gene expression in healthy and diseased individuals and human tissue

3. Investigate correlations between genotype and phenotype in clinical trial data

4. Study genetic and environmental factors involved in human disease

5. Display data visually using a graphical interface

6. Stratify clinical data into molecular subtypes of a specific disease

7. Collaborate across academic, government, and corporate research sectors. ${ }^{26}$

TranSMART can be thought of as a three-layer informatics system. The foundation is a collection of data domains. These could include clinical data, biomarker data, contextual data, and so on. Clinical data could include biochemical observations, patient outcomes, demographics, dosing, and so on.

The middle layer of tranSMART is the tranSMART Datamart. This is created from the foundational data after undergoing curation and loading with consideration paid to both information standards and syntactical standards.

The top layer is composed of analytical and modeling tools for mining information from the tranSMART Datamart as well as searching and browsing tools. The extensibility of the system allows for easily adding new data sets and incorporating new analytical tools using defined application programming interfaces (APIs). Analytical tools have been incorporated, such as a genome browser, analytics for such data as gene expression, and statistical tests such as

24 Ibid.

25 Shawn N. Murphy, Paul Avillach, Ricardo Delazzi, et al., Combining Clinical and Genomics Queries Using i2b2-Three Methods, 12(4) PLOS One (2017).

26 tranSMART Foundation, Realizing the Promise of Translational Biomedical Research, https://drive.google.com/file/d/ 0B8lizkKDeaKhMEJOdE5tVHR0YkE/view (last visited Jul. 29, 2020). 
ANOVA. New high-dimensional data types such as mass spec proteomics and metabolomics have also been added. ${ }^{27}$

\section{E. MOVING FORWARD}

Biological and health sciences follow a path whereby invention and data collection are followed by improvement into high-throughput approaches which create enormous amounts of data that are analyzed by evolving software. At the same time, data formats have moved from flat files to databases to data warehouses, and now to the creation of the Big Data branch of computer science.

In 1985 GenBank stored 5,204,420 bases of sequence and could be distributed on a set of floppy disks. In June 2020 it stored $4.27 \times 10^{11}$ bases of sequences. As computer speeds continue to increase, it will be possible to pull together larger amounts of data and potentially mimic the mind of the physician to aid in diagnosis - this is already in its infancy through clinical support tools.

Hopefully, an understanding of the present technologies in bioinformatics and medical informatics will provide an adequate backdrop to other chapters of this book for those in the law who are non-specialists in these technical fields. 